\title{
Alternativas para o estabelecimento de via aérea cirúrgica durante a pandemia de COVID-19.
}

\section{Alternatives for establishing a surgical airway during the COVID-19 pandemic.}

Daniel Souza lima, , ACBC-CE; Marcelo Fontenelle Ribeiro Junior, TCBC-SP2@i; Hélo Machado Vieira-Jr, TCBC-RJ3; Tércio de CAMPOS, TCBC-SP4; Salomone Di SAVERIO ${ }^{5}$

R E S U M O

Atualmente médicos e profissionais da saúde encontram-se frente a uma pandemia desafiadora causada por uma nova cepa denominada 2019 Novel Coronavírus (COVID-19). A infecção humana pelo COVID-19 ainda não tem o espectro clínico completamente descrito, bem como não se sabe com precisão o padrão de letalidade, mortalidade, infectividade e transmissibilidade. Não há vacina ou medicamento específico disponível. O tratamento é de suporte e inespecífico. No Brasil, assim como no restante do mundo o número de casos de COVID-19 tem crescido de maneira alarmante levando a um aumento do número de internações assim como da mortalidade pela doença. Atualmente os estados com maior número de casos são, respectivamente, São Paulo, Rio de Janeiro, Distrito Federal e Ceará. O objetivo deste trabalho é oferecer alternativas a fim de orientar cirurgiões quanto ao manejo cirúrgico das vias aéreas em pacientes com suspeita e/ou confirmação para infecção pelo COVID-19.

Descritores: Traqueostomia. Emergências. Pandemias.

Atualmente, médicos e profissionais da saúde encontram-se frente a pandemia desafiadora causada por nova cepa denominada 2019 Novel Coronavírus (COVID-19). A infecção humana pelo COVID-19 ainda não tem o espectro clínico completamente descrito, bem como não se sabe com precisão o padrão de letalidade, mortalidade, infectividade e transmissibilidade. Não há vacina ou medicamento específico disponível. O tratamento é de suporte e inespecífico'.

No Brasil, assim como no restante do mundo, o número de casos de COVID-19 tem crescido de maneira alarmante levando a aumento do número de internações e de mortalidade, pela doença. Atualmente os estados com maior número de casos são, respectivamente, São Paulo, Rio de Janeiro, Distrito Federal e Ceará?
Os profissionais de saúde que atuam na emergência necessitam adaptar a assistência aos pacientes atendidos com intuito de reduzir a contaminação e disseminação da doença. Até o início de março, a China notificou mais de 3300 profissionais de saúde infectados e pelo menos 22 mortes $^{3,4}$. Seguir diretrizes de proteção em procedimentos invasivos é essencial sempre, mas em especial neste momento assume relevante papel entre profissionais da emergência e cirurgiões.

O estabelecimento de via aérea definitiva está relacionado a difusão de aerossol pelo paciente, principalmente quando se usa oxigênio em alto fluxo. Por isso, devem-se utilizar cuidados que minimizem o risco de contaminação da doença. A ventilação com pressão positiva durante a ventilação não invasiva (VNI) ou quando se utiliza máscara

1 - Instituto Dr. José Frota e Universidade de Fortaleza UNIFOR, Serviço Cirurgia do Trauma e Departamento de Cirurgia - Fortaleza - CE - Brasil. 2 Hospital Moriah e IAMSPE, Cirurgia do Trauma e Programa de Pós Graduação - São Paulo - SP - Brasil. 3 - Complexo Hospital de Niterói, Serviço de Cirurgia Geral e do Trauma - Rio de Janeiro - RJ - Brasil. 4 - Faculdade de Ciências Médicas da Santa Casa de São Paulo, Cirurgia do Trauma - São Paulo - SP - Brasil. 5 - University of Insubria, University Hospital of Varese, ASST, Department of General Surgery - Sette Laghi - Lombardia - Itália. 
facial ou dispositivos supraglóticos é considerada de alto risco para geração de aerossóis, pela potencial ineficiência da vedação.

A laringocospia, a intubação traqueal e a broncoscopia irão produzir aerossol somente se houver estímulo de tosse ou se houver outros procedimentos associados (por exemplo, aspiração orotraqueal). Muitos destes eventos podem ser prevenidos com o bloqueio neuromuscular adequado e com o foco da equipe direcionado exclusivamente para o manejo das vias aéreas, sempre que possível.

O objetivo deste trabalho é oferecer alternativas a fim de orientar cirurgiões e outros profissionais quanto ao manejo cirúrgico das vias aéreas em pacientes com suspeita e/ou confirmação para infecção pelo COVID-19.

\section{Cricotireoidostomia ou traqueostomia (TQT) de Emergência (status desconhecido do COVID-19)}

Frente a atual pandemia, todos os pacientes atendidos nos setores de emergência devem ser considerados portadores do COVID-19, até que se prove o contrário. Pacientes que venham a apresentar sintomas respiratórios deverão, uma vez que não há até o presente momento um teste rápido para esta situação, ser tratados como infectados até a obtenção do resultado por meio de teste apropriado 5 .

O manejo da via aérea de maneira geral deve SER: Seguro, Exato e Rápido. Seguro para os profissionais e pacientes; exato e preciso evitando técnicas não confiáveis, desconhecidas ou repetidas; rápido evitando atraso não só para a indicação como para realização do procedimento ${ }^{6}$. Figura 1.

\section{TQT eletiva}

Testar o paciente para COVID-19 antes do procedimento

A indicação precisa e a avaliação correta da necessidade do procedimento devem ser consideradas para que se evite riscos desnecessários de disseminação da doença.

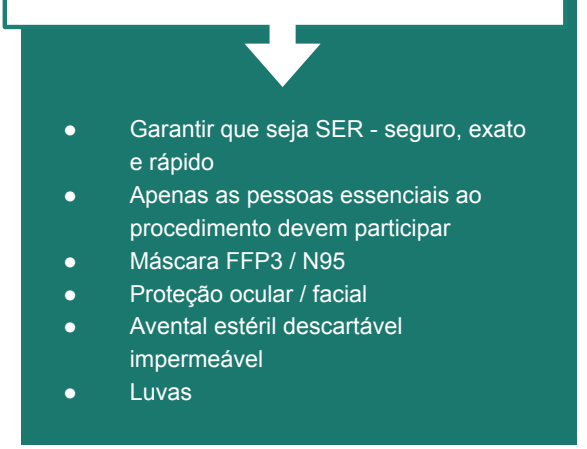

\section{Crico ou TQT de Emergência}

Em caso de falha da IOT

A crico cirúrgica é a primeira opção. A por punção se reserva para casos de impossibilidade na obtenção de crico cirúrgica

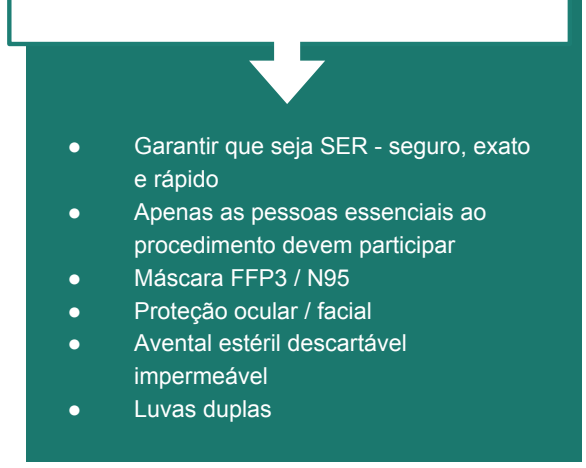

\section{Cuidados pós traqueostomia}

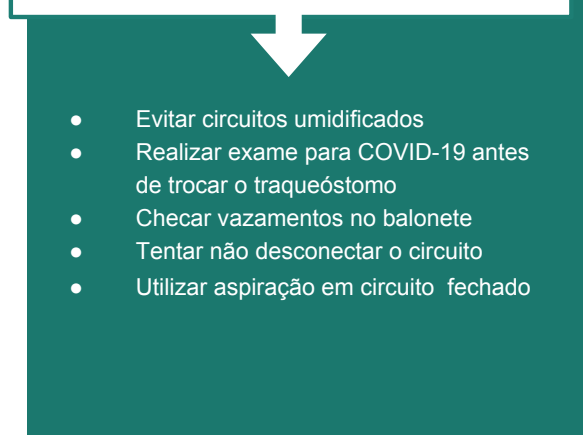

Figura 1. Abordagem cirúrgica das vias aéreas. 
Alternativas para obstrução das vias aéreas ${ }^{5}$

- A intubação orotraqueal (IOT) deve ser sempre a primeira via de eleição, evitando-se a via aérea cirúrgica de maneira intempestiva. Portanto, procure seguir sempre as orientções para o manejo de via aérea difícil;

- Evite o uso de oxigênio de alto fluxo;

- O médico que irá executar o manejo da via aérea deve ser preferencialmente experiente para maximizar o sucesso da primeira tentativa de IOT;

- Em caso de suspeita de via aérea difícil, é recomendável o acompanhamento de profissional com experiência em vias aéreas cirúrgicas;

- Minimize o número de pessoas à beira do leito. Há a recomendação para 3 profissionais: o médico responsável pelo procedimento, um assistente ( $p$. ex: enfermeiro) e um administrador de medicações/monitorização (p.ex:: técnico de enfermagem) ${ }^{6}$.

A cricotireoidostomia cirúrgica é a preferível frente à cricotireoidostomia por punção por ser mais eficaz e diminuir a disseminação de aerossóis. A técnica por punção fica reservada para casos de exceção nos cenários de emergência.

Procura-se identificar e palpar a membrana cricotireoide, com dois cenários possíveis ${ }^{5}$, conforme demonstrado na tabela 1.

\section{Traqueostomia Eletiva}

O tempo máximo a que um paciente pode ser mantido em IOT, sem risco de lesão traqueal, é controverso. Há relatos de benefícios entre pacientes submetidos à TQT, podendo esta, de acordo com diferentes definições ser realizada com 2, 7 ou 10 dias de ventilação mecânicå .
Considerando-se que a traqueostomia é um procedimento eletivo, recomenda-se a confirmação diagnóstica prévia do COVID-19 e estabilização clínica, pois trata-se de procedimento de alto risco pela capacidade de geração de aerossóis ${ }^{5}$.

A indicação precisa e a avaliação correta da necessidade do procedimento devem ser consideradas para que se evitem riscos desnecessários de disseminação da doença.

Se o teste for negativo para COVID-19, realize o procedimento operacional padrão (máscara cirúrgica resistente a líquidos, bata cirúrgica, luvas e proteção para os olhos).

\section{Procedimento operatório padrão para traqueostomia em paciente positivo COVID-19/ status desconhecido ${ }^{5}$}

- Especialistas que executam a sedoanalgesia e o procedimento cirúrgico, devem garantir que este seja seguro, exato e rápido (SER).

- Reduza os membros da equipe para apenas os essenciais.

- Preparação e paramentação:

1. Use a máscara FFP3/N95;

2. Utilize proteção ocular / facial sempre que realizar TQT ou trocar um tubo de TQT devido ao risco de secreções respiratórias ou líquidos corporais. Uma das seguintes opções é adequada:

a. máscara cirúrgica com viseira integrada;

b. protetor facial completo / viseira;

3. Utilize avental descartável resistente a líquidos/soluções (impermeável). Se for usado avental permeável, utilize avental de plástico descartável por baixo. O avental deve ser esterelizado, de mangas longas, punho de malha ou elásticoeabertura posterior. Além disso, 
Tabela 1. Cenários possíveis na cricotireoidostomia de urgência.

\begin{tabular}{|c|c|}
\hline 1 - Membrana cricotireóidea palpável & 2 - Membrana cricotireóidea não palpável \\
\hline - realize incisão cutânea transversa ou longitudinal & - realize incisão cutânea longitudinal ampla \\
\hline $\begin{array}{c}\text { - faça incisão transversa na membrana } \\
\text { cricotireóidea }\end{array}$ & $\begin{array}{l}\text { - localize a membrana cricotireóidea por dissecção } \\
\text { romba com os dedos, estabilizando a laringe }\end{array}$ \\
\hline $\begin{array}{l}\text { - proceda com a dilatação utilizando pinça ou cabo } \\
\text { do bisturi }\end{array}$ & $\begin{array}{c}\text { - faça incisão transversa na membrana } \\
\text { cricotireóidea }\end{array}$ \\
\hline $\begin{array}{l}\text { - tenha como opção utilizar o bougie para aposição } \\
\text { do traqueóstomo }\end{array}$ & $\begin{array}{c}\text { - proceda com dilatação utilizando pinça ou cabo } \\
\text { do bisturi }\end{array}$ \\
\hline - realize aposição do traqueóstomo & $\begin{array}{c}\text { - opte por utilizar o bougie para aposição do } \\
\text { traqueóstomo }\end{array}$ \\
\hline $\begin{array}{l}\text { - garanta a confirmação preferencialmente com } \\
\text { capnógrafo, se disponível }\end{array}$ & - realize a aposição do traqueóstomo \\
\hline \multirow[t]{2}{*}{ - Realize a ausculta7 } & $\begin{array}{c}\text { - garanta a confirmação preferencialmente com } \\
\text { capnógrafo, se disponível. }\end{array}$ \\
\hline & - Realize a ausculta \\
\hline
\end{tabular}

deve ser confeccionado com material de boa qualidade, não alergênico e resistente. Deve proporcionar barreira antimicrobiana efetiva, permitir a execução de atividades com conforto e estar disponível em vários tamanhos ${ }^{9}$;

4. As luvas devem ser adequadas para permitir a manipulação manual com conforto e sem limitar a destreza. Considere usar "duas luvas". Em alguns países existe a disponibilidade de luvas com conteúdo de biogel que exterioriza e sinaliza quando há perfuração.

- Utilizar traqueóstomo convencional, evitando modelos frenestrados;

- Todo esforço deve ser feito para não perfurar o balonete do tubo endotraqueal ao realizar a TQT;

- Realizar avanço inicial do tubo endotraqueal antes da janela da TQT ser executada para reduzir eliminação de aerossóis;

- Se possível, interrompa a ventilação enquanto a janela da traqueia estiver sendo realizada e verifique se o balonete já está inflado antes de reiniciar a ventilação;

- A ventilação é interrompida antes da inserção do traqueóstomo, realize a colocação rápida e precisa do traqueóstomo com insuflação imediata do balonete;

- Confirme o posicionamento preferencialmente com capnografo, se disponível;

- Verifique se não há vazamentos e se o traqueóstomo está bem fixado;

- Um filtro tipo HME (Heat and moisture exchanger) ou HEPA deve ser obrigatoriamente colocado no traqueóstomo para reduzir a exposição do vírus, caso haja desconexão.

Uma alternativa proposta, tanto nos procedimentos eletivos como de urgência, é usar um extensor obstruído pelo êmbolo de uma seringa e, em seguida, usar imediatamente uma pinça para obstrução, remoção do êmbolo e conexão ao sistema de ventilação mecânica (figura 2). 


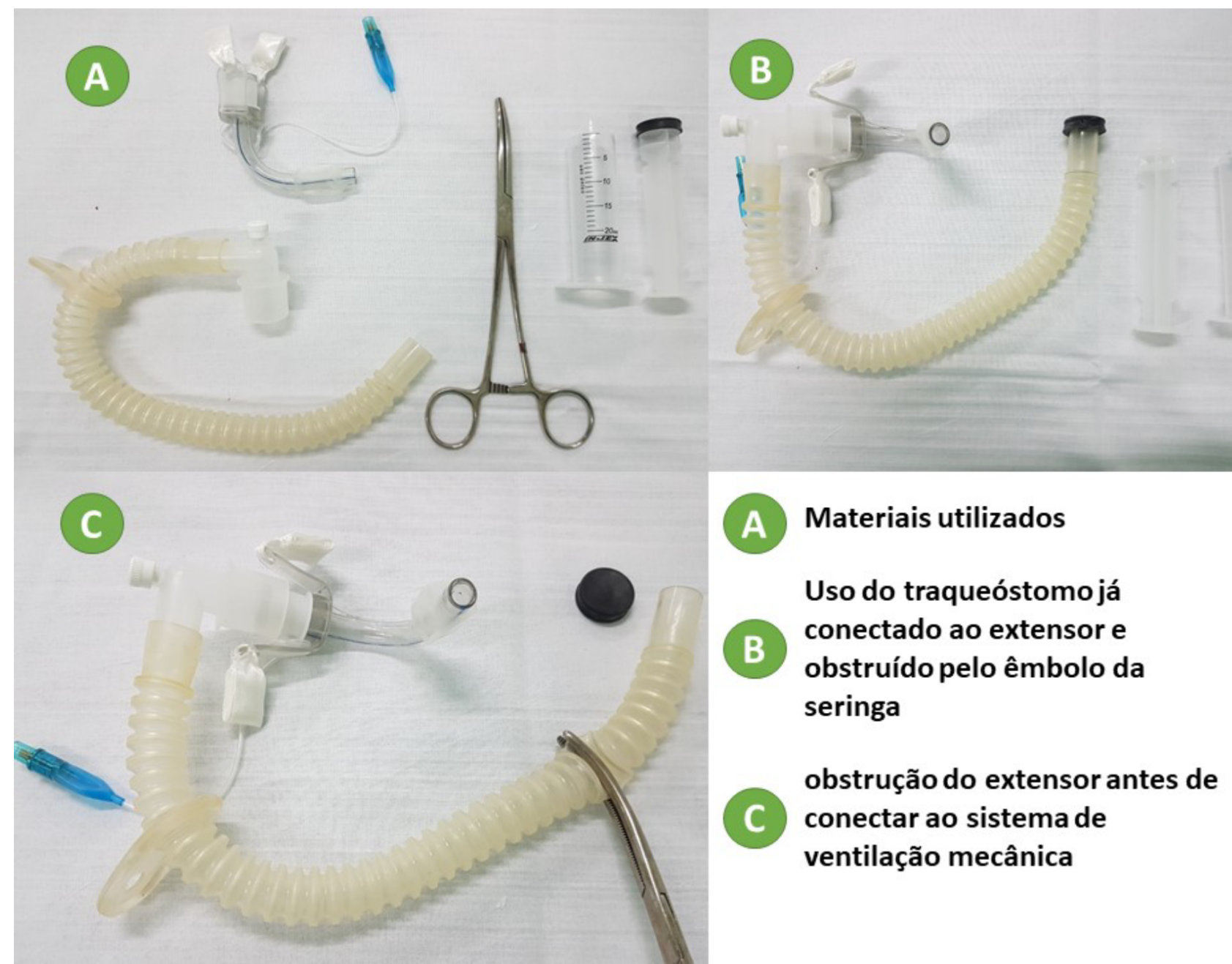

Figura 2. Adaptações para reduzir o risco de exposição a aerossóis.

\section{Cuidados pós-traqueostomia}

- O Royal College of Anaesthetists sugere evitar circuitos umidificados, pois teoricamente esse reduzirá os riscos de contaminação da sala se houver desconexão inesperada do circuito;

- Evite trocar o traqueóstomo até que o paciente apresente exame negativo para COVID-19;

- Verifique a manutenção da insuflação do balonete e se há vazamentos

- Faça todos os esforços para não desconectar o circuito;
- Somente a aspiração em circuito fechado deve ser usada.

\section{Considerações importantes}

O equipamento de proteção individual é apenas parte de um sistema para evitar a contaminação e infecção dos profissionais de saúde. Além do equipamento de proteção, procedimentos como descontaminação de superfícies e equipamentos, minimização do contato desnecessário com o paciente e superfícies ao redor do local de realização do procedimento e gerenciamento cuidadoso de resíduos são essenciais para a redução de riscos ${ }^{5}$. 
O vírus pode permanecer viável no ar por período prolongado e em superfícies não absorventes por muitas horas e até dias ${ }^{10}$. No entanto, a remoção de partículas infecciosas após um procedimento de geração de aerossol depende da ventilação mecânica / natural e da troca de ar, por hora, na sala. Estima-se que uma única mudança de ar remova $63 \%$ dos contaminantes transportados pelo ar. Com base nisso, o tempo mínimo considerado para a limpeza do ambiente após a liberação do aerossol é de 20 minutos ${ }^{6,11}$.

Atualmente classificam-se os procedimentos sobre as vias aéreas de acordo com o risco conforme abaixo (expresso em ordem decrescente) ${ }^{10}$ :

Intubação traqueal;

Traqueostomia/Cricotiroidostomia;
Ventilação não invasiva (VNI);

Máscara de ventilação.

\section{CONCLUSÕES}

No lado oposto do isolamento social, estão os profissionais de saúde na linha de frente do enfrentamento do COVID-19, com riscos maiores de infecção. A garantia de equipamentos de proteção individual deve ser priorizada, associada a adaptações da assistência e dos procedimentos cirúrgicos com intuito de reduzir o risco de infecção. Procedimentos com exposição de aerossóis do paciente, como a cricotiroidostomia e traqueostomia, devem ser adaptados e, exigem a educação dos médicos emergencistas e cirurgiões. Os profissionais de saúde são os recursos mais valiosos neste momento.

\title{
A B S T R A C T
}

\begin{abstract}
Currently doctors and health professionals are facing a challenging pandemic caused by a new strain called 2019 Novel Coronavirus (COVID-19). Human infection with COVID-19 does not yet have the clinical spectrum fully described, and the pattern of lethality, mortality, infectivity and transmissibility is not known with precision. There is no specific vaccine or medication available. Treatment is supportive and nonspecific. In Brazil, as in the rest of the world, the number of COVID-19 cases has grown alarmingly, leading to an increase in the number of hospitalizations as well as in mortality from the disease. Currently, the states with the highest number of cases are, respectively, São Paulo, Rio de Janeiro, Distrito Federal and Ceará. The objective of this work is to offer alternatives in order to guide surgeons regarding the surgical management of the airways in patients with suspicion and / or confirmation for COVID-19 infection.
\end{abstract}

Keywords: Emergencies. Tracheostomy. Pandemics.

\section{REFERÊNCIAS}

1. Guan WJ, Ni ZY, Hu Y, Liang WH, Ou CQ, He JX, Liu L, Shan H, Lei CL, Hui DSC, Du B, Li L, Zeng G, Yuen KY, Chen RC, Tang CL, Wang T, Chen PY, Xiang J, Li SY, Wang JL, Liang ZJ, Peng YX, Wei L, Liu Y, Hu YH, Peng P, Wang JM, Liu JY, Chen Z, Li G, Zheng ZJ, Qiu SQ, Luo J, Ye CJ, Zhu SY, Zhong NS; China Medical Treatment Expert Group for Covid-19. Clinical Characteristics of Coronavirus Disease 2019 in China. N Engl J Med, [publicação online];2020 [acesso em 2020 Mar 25]. Disponível em: https://www.nejm. org/doi/full/10.1056/NEJMoa2002032
2. Ministério da Saúde (BR). Coronavírus: 25 mortes e 1.546 casos confirmados. [publicação na web]; 2020 [acesso 2020 Mar 22]. Disponível em: https://www.saude.gov.br/ noticias/agencia-saude/46573-coronavirus-25-mortes-e1-546-casos-confirmados

3. Organização das Nações Unidas. OMS alerta sobre contaminação de profissionais de saúde por Covid-19. [publicação na web]; 2020 [acesso 2020 Mar 19]. Disponível em: https://news.un.org/pt/ story/2020/02/1704211

4. The Lancet. COVID-19: protecting health-care workers. Lancet, [publicação online]; 2020 [acesso 2020 Mar 22]. Disponível em: https://www.thelancet.com/ journals/lancet/article/PIIS0140-6736(20)30644-9/fulltext 
5. Harrison L, Ramsden J, Winter S, Rocke J, Heward E. Guidance for Surgical Tracheostomy and Tracheostomy Tube Change during the COVID-19 Pandemic. 2020.[publicação na web]; 2020 [acesso 2020 Mar 20]. Disponível em: https://www.entuk. org/tracheostomy-guidance-during-covid-19pandemic

6. The Faculty of Intensive Care Medicine and Intensive Care Society and Association of Anaesthetists and Royal College of Anaesthetists. COVID-19: Airway management principles. [publicação na web]; [acesso 2020 Mar 19]. Disponível em: https:// icmanaesthesiacovid-19.org/airway-management

7. Cheung JC, Ho LT, Cheng JV, Cham EYK, Lam KN. Staff safety during emergency airway management for COVID-19 in Hong Kong. Lancet Resp Med, [publicação online]; 2020 [acesso 2020 Mar 20]. Disponível em: https://www.thelancet.com/journals/ lanres/article/PIIS2213-2600(20)30084-9/fulltext

8. Cardoso L, Simoneti FS, Camacho EC, Lucena RV, Guerra AF, Rodrigues JMS. Intubação orotraqueal prolongada e a indicação de traqueostomia. Rev. Fac. Ciênc. Méd. Sorocaba. 2014;16 (4):170-73.

9. Ministério da Saúde do Brasil. Protocolo de manejo clínico para o novo coronavírus (2019-nCOV). [publicação na web]; 2020 [acesso em 2020 Mar 19]. Disponível em: https://portalarquivos2.saude.gov. br/images/pdf/2020/fevereiro/11/protocolo-manejocoronavirus.pdf
10. van Doremalen N, Bushmaker T, Morris DH, Holbrook MG, Gamble A, Williamson BN, et al. Aerosol and surface stability of HCoV-19 (SARS-CoV-2) compared to SARS-CoV-1. N Engl J Med, [publicação online];2020 [acesso em 2020 Mar 19]. Disponível em: https://www.nejm.org/doi/full/10.1056/ NEJMc2004973

11. Department of Health and Social Care, Public Health Wales, Public Health Agency Northern Ireland, Health Protection Scotland and Public Health England. COVID-19: guidance for infection prevention and control in healthcare settings. [publicação na web]; 2020 [acesso em 2020 Mar 25]. Disponível em: https://assets.publishing.service.gov.uk/government/ uploads/system/uploads/attachment_data/ file/874316/Infection_prevention_and_control_ guidance_for_pandemic_coronavirus.pdf

Recebido em: 30/03/2020

Aceito para publicação em: 01/04/2020

Conflito de interesses: Não

Fonte de financiamento: Não

\section{Endereço para correspondência:}

Daniel Souza Lima

E-mail: souzadl@hotmail.com

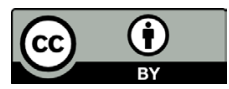

